\title{
Pseudo-transient computational fluid dynamics analysis of an underbonnet compartment during thermal soak
}

\author{
M Franchetta ${ }^{1 *}$, K O Suen ${ }^{2}$, and T G Bancroft ${ }^{3}$ \\ ${ }^{1}$ Vehicle Thermal Systems, Ricardo UK, Midlands Technical Centre, Leamington Spa, UK \\ ${ }^{2}$ Department of Mechanical Engineering, University College London, London, UK \\ ${ }^{3}$ Ricardo Inc., Detroit Technical Center, Detroit, USA
}

The manuscript was received on 9 February 2007 and was accepted after revision for publication on 22 June 2007.

DOI: 10.1243/09544070JAUTO555

\begin{abstract}
Underbonnet simulations are proving to be crucially important within a vehicle development programme, reducing test work and time-to-market. While computational fluid dynamics (CFD) simulations of steady forced flows have been demonstrated to be reliable, studies of transient convective flows in engine compartments are not yet carried out owing to high computing demands and lack of validated work. The present work assesses the practical feasibility of applying the CFD tool at the initial stage of a vehicle development programme for investigating the thermally driven flow in an engine bay under thermal soak.

A computation procedure that enables pseudo time-marching CFD simulations to be performed with significantly reduced central processing unit (CPU) time usage is proposed. The methodology was initially tested on simple geometries and then implemented for investigating a simplified half-scale underbonnet compartment. The numerical results are compared with experimental data taken with thermocouples and with particle image velocimetry (PIV).

The novel computation methodology is successful in efficiently providing detailed and timeaccurate time-dependent thermal and flow predictions. Its application will extend the use of the CFD tool for transient investigations, enabling improvements to the component packaging of engine bays and the refinement of thermal management strategies with reduced need for in-territory testing.
\end{abstract}

Keywords: CFD, transient, underbonnet, natural convection, thermal management, PIV

\section{INTRODUCTION}

Underbonnet thermal management has become an increasingly important aspect of vehicle design as increased engine power and cooling requirements, styling restrictions, encapsulation features, and space limitations have presented engineers with a difficult problem to solve. To compound the situation, there is increasing pressure to reduce product development times in order to respond faster to market trends. The need for a cost-effective solution that satisfies system performance targets requires a detailed under-

* Corresponding author: Vehicle Thermal Systems, Ricardo UK, Southam Road, Radford Semele, Leamington Spa, Warwickshire CV31 1FQ, UK. email: matteo.franchetta@ricardo.com standing of the flow and thermal interactions in the engine bay in order to optimize system architecture and thermal management strategies.

Thermal issues in the underbonnet space have traditionally been investigated via extensive interritory and wind tunnel tests carried out on successive generations of prototypes. To save on the expensive and time-consuming test work, numerical analyses have been introduced, particularly in the initial stages of vehicle development programmes. Underbonnet simulations with computational fluid dynamics (CFD) have proved to be an attractive alternative to empirical investigations. However, they have only been validated for steady forced flow conditions $[\mathbf{1}, \mathbf{2}]$. Transient analyses of processes with a long timescale, such as the thermal evolution of the 
buoyant airflow under the bonnet during thermal soak, are still unfeasible owing to high computing requirements (a typical transient analysis of a 10 minute period would take several weeks to complete, when performed even on a central processing unit (CPU) cluster architecture).

Thermal or heat soak refers to the build-up of temperature under the bonnet after high-speed/load driving, followed by engine 'key-off'. As the fans and cooling system stop operating, the high residual heat from the turbocharger (and close-coupled catalyst) is dissipated with difficulty from the engine bay. With reduced air movement (only driven by natural convection) the underbonnet components are subjected to high thermal loads, particularly if located in the vicinity of the exhaust parts.

Previous attempts to reduce the processing time for transient CFD computations have failed to demonstrate the saving in CPU runtime needed to make them feasible on the computing resources available to a typical engineering analysis group. The attempts have focused on reducing the complexity of the modelled geometries, implementing empirical expressions as boundary conditions [3], adopting simplified numerical models [4], and integrating more efficient numerical algorithms into the CFD solver [5]. Reductions in the CPU runtime up to 50 per cent have been achieved (arguably sufficiently), but often at the expense of marked losses in prediction accuracy.

The present work was aimed at developing a new approach for solving heat soak processes in engine bays. A computational methodology was devised and implemented in the commercial CFD code VECTIS. The objective was to deliver accurate timedependent predictions with substantially reduced computing time when compared with a standard, fully conservative, transient simulation.

The parameters affecting the performance of the novel methodology were investigated and refined systematically by comparing the flow and thermal predictions of simple test cases with their corresponding fully transient solution. The overall accuracy and efficiency of the computation were then assessed by correlating the transient predictions of a simplified half-scale underbonnet model with measurements.

Particle image velocimetry (PIV) was used to acquire quantitative information on the flow velocity and to provide a qualitative insight into the flow patterns developing in the underbonnet model. In addition, temperature measurements of surfaces and air planes were made with thermocouples. The experimental investigation enabled accurate data to be collected for comparison with the CFD predictions and is expected to partly compensate for the lack of validation studies in the literature on natural convective flows in engine compartments.

\section{PROPOSED METHODOLOGY}

The fundamental limitation that makes CFD transient computations inherently difficult is the requirement for timesteps of the order of $1 / 1000$ th of a second to ensure numerical accuracy and stability for the flow momentum solution. During thermal soak, the high heat capacity of underbonnet components combined with the weak heat transfer associated with natural convection results in a period of time up to 30 minutes during which the temperature and the flow fields in the engine compartment are continually changing. The magnitude of difference between these two timescales makes time-dependent simulations inherently resource intensive and currently unfeasible to be completed within an acceptable industrial timeframe.

The proposed methodology circumvents the limitation on the flow momentum solution by 'freezing' the flow field, i.e. pausing the solution of all governing equations except for the temperature. By freezing the airflow the analysis is freed from having to run very small timesteps and the thermal solution can quickly advance in time. A time-accurate flow field, based on the temperatures of the underbonnet components at the end of the frozen period, is then updated/re-established by 'un-freezing' the flow and fully solving for conservation of momentum. The procedure is then repeated until the time period of interest is fully covered.

This piecewise, non-conservative, or 'pseudotransient', solution approach is schematically shown in Fig. 1. Long periods of time allocated to 'frozen flow' solution processes alternate with short periods of time allocated to 'unfrozen flow' processes.

\section{TESTING OF SOLVER PARAMETERS}

The methodology was implemented in the Reynoldsaveraged Navier-Stokes (RANS) software VECTIS, which was set to perform the computations in conjugate heat transfer (CHT) mode (i.e. simultaneous solution of the temperature field in coupled solid and fluid domains [6]). Two variants of the proposed approach, henceforth referred to as the UPDATE and the SWITCH variants, were devised and tested. These differed in the type of numerical solver employed for the flow field update, i.e. transient or steady state 


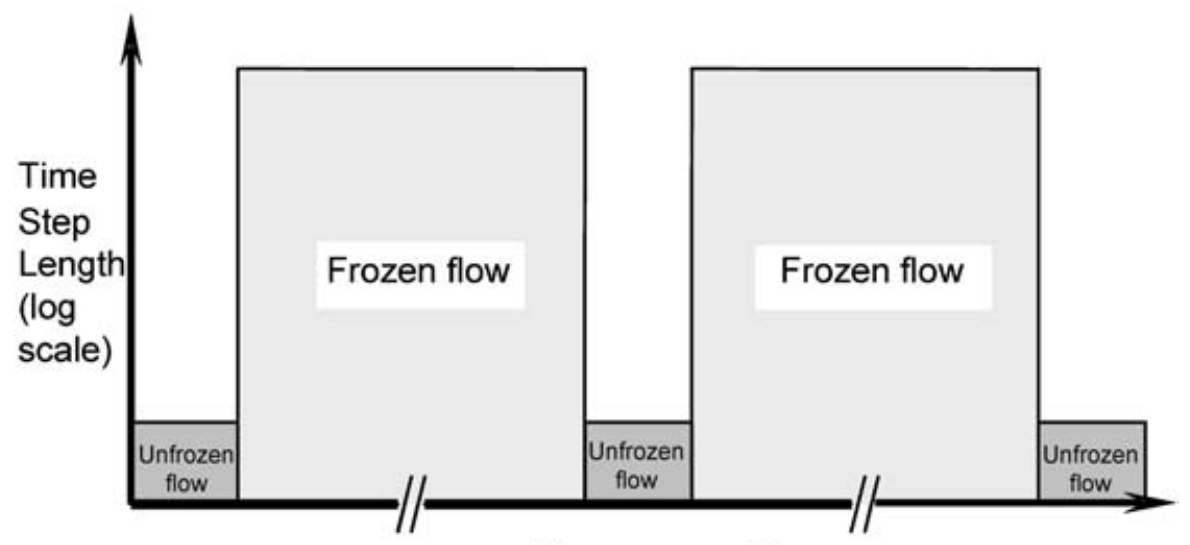

Time progression

Fig. 1 Solution scheme (length of frozen flow periods and corresponding time steps are several orders of magnitude larger than that of unfrozen flow periods)

respectively. Both variants were set to start with a 'full' computation (i.e. transient or steady solution of the complete set of Navier-Stokes equations). They then progressed in cycles, with each cycle comprising a computation for the fast advancement in time (referred to as 'fast transient computation' in Fig. 2) and a computation for updating the flow transport ('full transient' or 'steady state' computation).

Figure 2 lists the governing equations solved by the individual CFD solution processes. A program was specifically written in $\mathrm{Java}^{\mathrm{TM}}$ to automate fully the alternation between computations.

A model with a relatively simple geometry was employed to investigate the effects of different solver parameters on the efficiency and accuracy of the proposed CFD transient strategies, for refining and optimizing the calculation procedures. The model consisted of a hollow block $\left(100 \times 100 \times 100 \mathrm{~mm}^{3}\right.$, wall thickness $10 \mathrm{~mm}$ ) positioned at the centre of a computational chamber $\left(500 \times 500 \times 100 \mathrm{~mm}^{3}\right)$
(Fig. 3). The small and identical width of the block and the chamber (i.e. $100 \mathrm{~mm}$ ) represented a less computationally demanding configuration, which enabled a reference full transient simulation to be carried out, providing the necessary data for comparison. The block participated in the CHT simulation as a solid model with four of its surfaces thermally conjugated with the airflow domain (i.e. set to exchange local heat transfer coefficients and temperature values) and the remaining two (vertical surfaces) specified as 'zero gradient' (slip + adiabatic walls). The initial boundary conditions were set up so as to simulate a transient cooling process.

Several solver parameters were observed to affect the performance of the two transient procedures:

(a) number of cycles performed (i.e. number of flow updates);

(b) number and length of the timesteps in the fast transient computations (frozen flow periods);

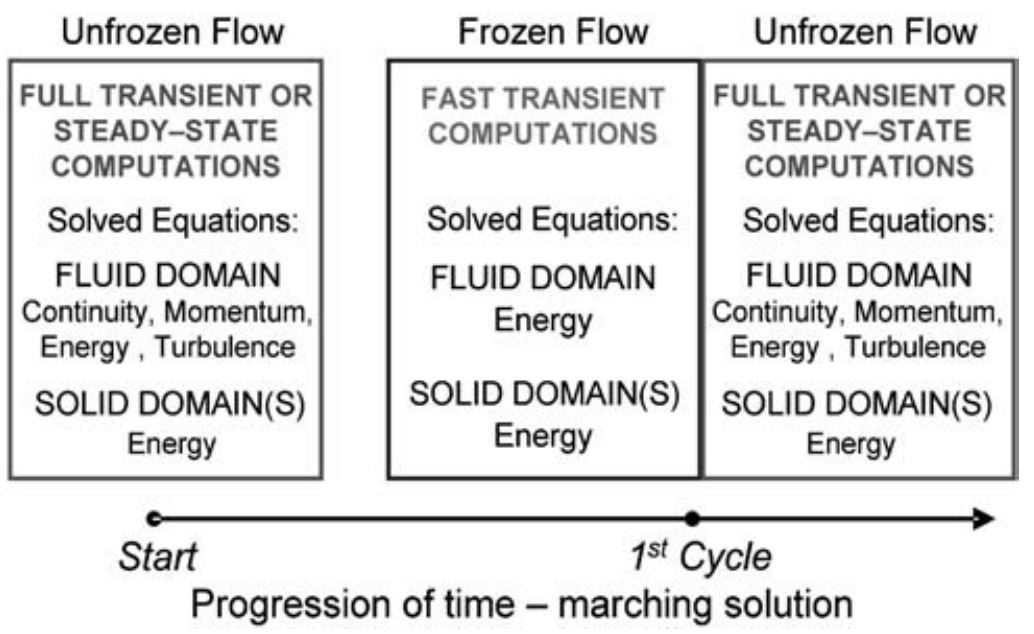

Fig. 2 Solver scheme 


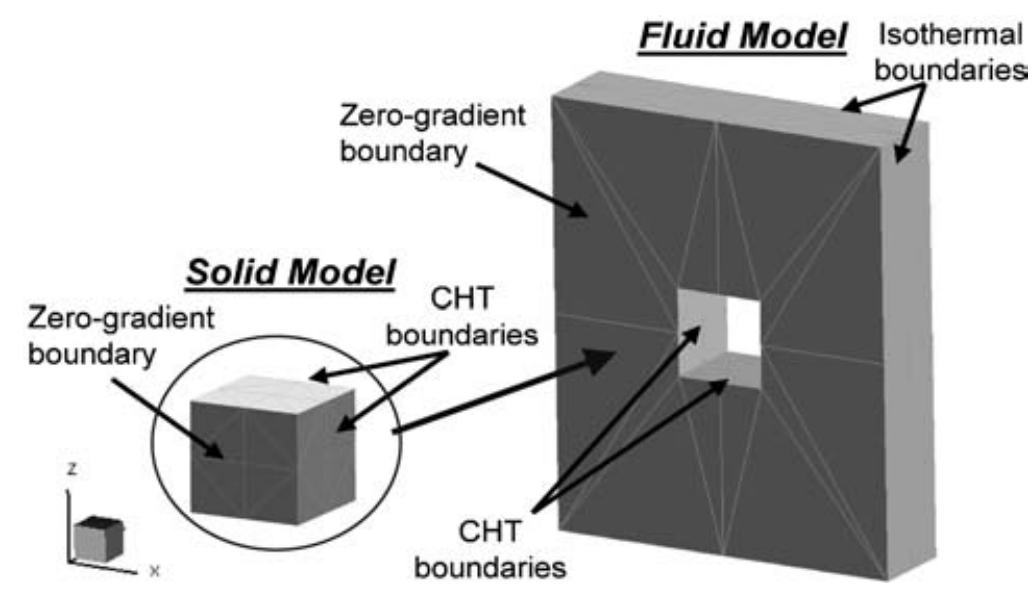

Fig. 3 Test geometry employed for testing and refining the solver parameters of the proposed methodologies. The solid and fluid CHT models are shown separately

(c) number and length of the timesteps in the full transient or steady state computations (unfrozen flow periods).

The most significant cause of discrepancy with the reference full transient predictions was identified as the number of flow solution updates performed, i.e. the total number of cycles performed to resolve the time-dependent timeframe. Tests were conducted whereby the number of methodology cycles was progressively increased; as a consequence, the number of time steps in the frozen flow periods had to reduce in order to discretize the same time period of interest (set at $1500 \mathrm{~s}$ ).

The optimal number of methodology cycles was found to be 300 for the investigated model, and the length of the fast transient runs was set to $5 \mathrm{~s}$ (1500 s/300 = $5 \mathrm{~s}$ ), with steps advancing the solution by $1 \mathrm{~s}$ each. Additional tests on different geometries established that the timestep length should generally be set in the range of 100 to 1000 times larger than its optimal length in a corresponding fully conservative transient computation (assuming an identical convergence index is set on the implicit solver of the N-S equations). Further analysis also established that a similar prediction accuracy is obtained when the length of the frozen flow periods is increased after approximately the first 30 per cent of the computation. Indeed, when the variation of momentum between two successive unfrozen-flow periods becomes relatively small and the numerical solution more stabilized, a larger number of steps can be taken to advance the thermal solution during the frozen-flow periods. The consequent reduction in the number of computations results in a further decrease of the overall CPU turnaround time required for the simulation.
For updating the flow momentum, it emerged that a larger number of transient timesteps (or steady iterations) improves the accuracy of the flow field predictions, albeit at the expense of increased CPU runtime. However, the study also revealed that a compromise between accuracy and runtime can be achieved by reducing the number of numerical iterations during the unfrozen-flow computations (i.e. the number of transient timesteps or steady iterations) while increasing the total number of methodology cycles (the frequency of flow updates). The processing time would in fact decrease without any evident deterioration in the quality of the CFD predictions. The accuracy of the simulation was therefore found to benefit more from a larger number of 'short' momentum updates than from a smaller number of 'long' ones.

The reductions of the timestep length (for the transient computations) and convergence index were found not to be a key factor for improving the performance of the methodology. Indeed, a reduction in timestep length was found to increase the CPU effort greatly with relatively small benefits to the numerical stability and prediction accuracy. The solution step and convergence index should therefore be set equal to those of a standard transient computation.

During the parametric investigations, a problem in the computational procedure based on steady state flow updates was identified. During the steady processes, the solution of both the solid and the airflow domains were observed to tend towards thermal equilibrium. This was not desirable since the steady runs were only required to update the momentum transport without advancing the time-dependent solution. To overcome the problem, the steady thermal solution of solid models (energy equation) was frozen 
in the simulation while maintaining the CHT links with the fluid domain (this was implemented in VECTIS by not specifying the material properties of the solid components).

The results of the tests indicated that, overall, both methodologies provided equally satisfactory predictions when compared with the reference simulation. Figures 4(a) and (b) compare the combined average data discrepancy calculated at 500, 1000, and 1500 seconds. The vertical and horizontal components of the air velocity were predicted by both the UPDATE and the SWITCH variants to be within 50 per cent of the reference magnitudes. This was regarded as satisfactory considering the very small air velocities in the model and the numerical oscillations observed to affect the flow solution (owing to the use of a relatively large timestep length of $0.01 \mathrm{~s}$ for solving the unfrozen flow periods); the average absolute discrepancy of the two sets of results was only of the order of $10^{-4} \mathrm{~m} / \mathrm{s}$. An exceptionally good agreement was obtained between temperature predictions, with discrepancies ranging from 0.1 to 0.6 per cent. The SWITCH methodology, based on steady state flow updates, appeared to be the more efficient, with a saving in processing time in excess of 90 per cent and a reduction in disk storage space of about 12 per cent compared with the corresponding fully conservative transient computation (Fig. 4(c)).

\section{APPLICATION TO UNDERBONNET}

\subsection{Computational model}

The case study used to test the applicability of the developed procedure was based on the half-scaled underbonnet model shown in Fig. 5. It consisted of a slotted compartment $(0.57 \mathrm{~m} \times 0.56 \mathrm{~m}$ base area) with (a)

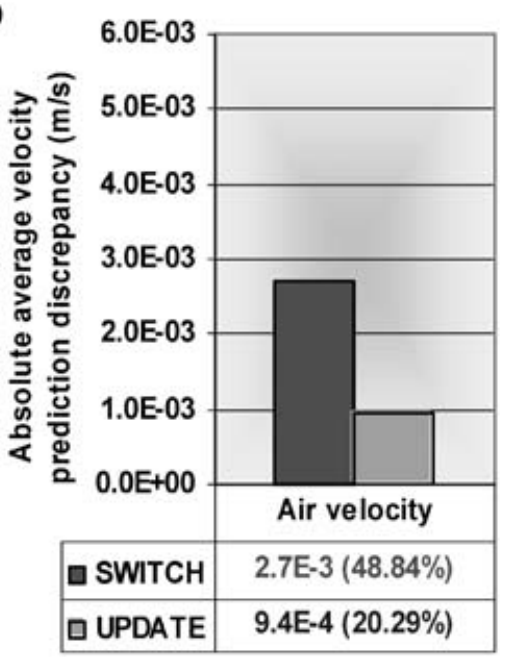

(b)

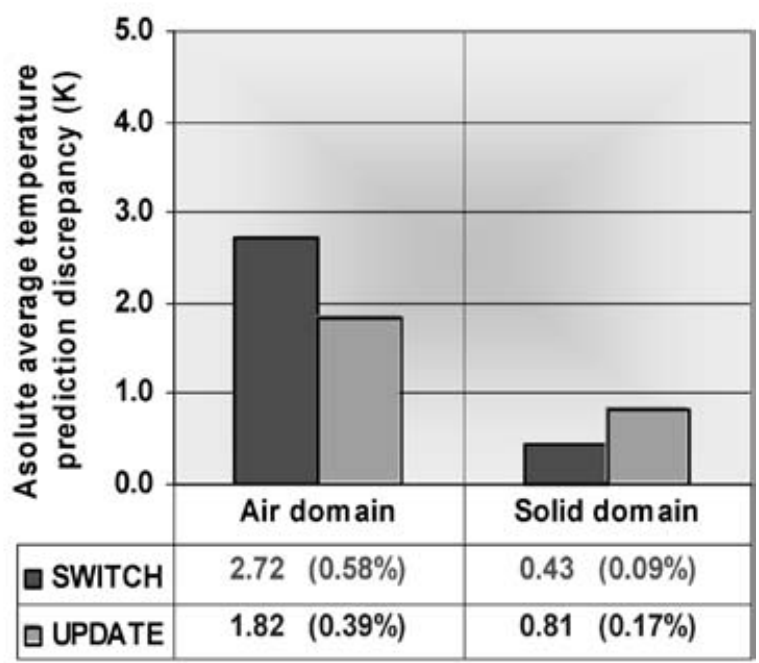

(c)

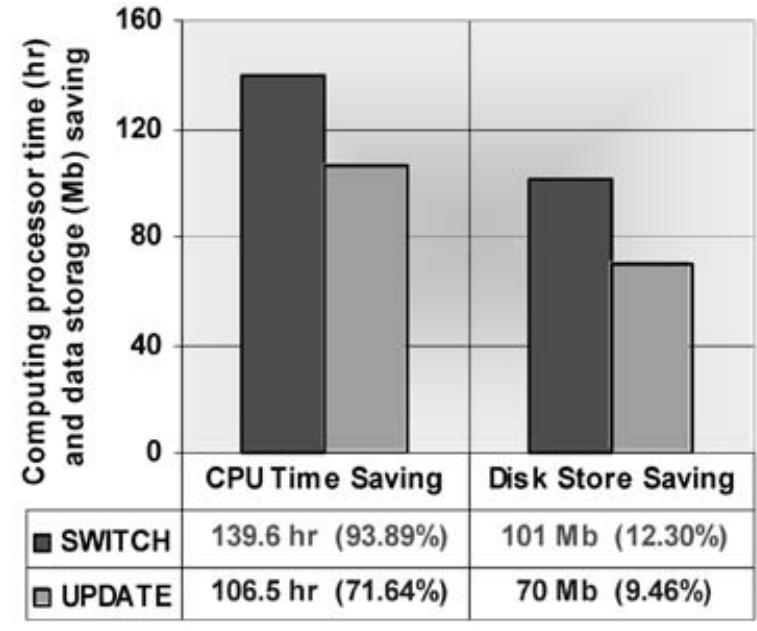

Fig. 4 Absolute average data discrepancy (a, b) and hardware processing saving (c) for tested methodology variants relative to corresponding full transient simulation (percentage differences given in brackets) 


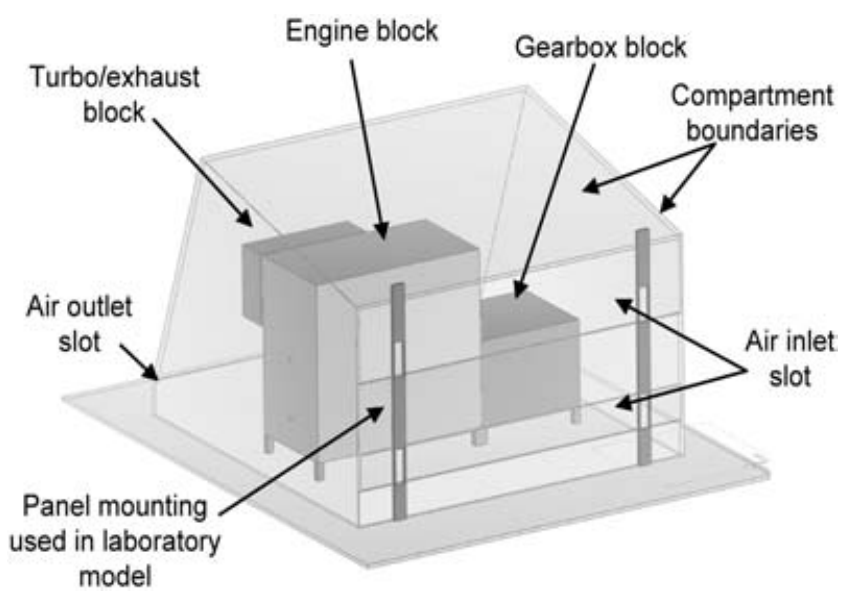

Fig. 5 Simplified underbonnet model used to test the applicability of proposed transient procedure

inlet and outlet openings set respectively in correspondence to the front and rear-end vertical surfaces. It incorporated metal blocks representing in scaled size a typical engine, gearbox, and turbocharger/ exhaust system. The compartment was placed in a computational chamber to obviate the need of applying specific boundary conditions at the openings to ambient.

The model was simulated for approximately 30 minutes while cooling down from an initial average engine-gearbox temperature of $120^{\circ} \mathrm{C}$ and a mean turbocharger/exhaust temperature of $360{ }^{\circ} \mathrm{C}$, which were previously established with a steady state simulation [7]. The analysis was aimed at replicating the transient thermal condition experienced by an engine compartment when in thermal soak.

\subsection{Experimental measurements}

The accuracy of the transient solution procedure was assessed by comparing the simulation results with laboratory measurements. The underbonnet laboratory rig (Fig. 6), was made of high-temperatureresistant glass to allow optical access and incorporated flat surface blocks fabricated from cast iron and aluminium. The geometry was identical in shape/size to the computational model. Cylindrical cartridge heaters, regulated by bespoken temperature controllers, were embedded in the blocks in order to provide the required heat input for establishing a steady thermal state before the transient cooling.

The experimentations consisted of air and block surface temperature measurements taken with $\mathrm{T}$ - and K-type thermocouples logged to a data acquisition board. The sensors were appropriately shielded from the thermal radiation of the block surfaces.

Figure 7 shows a schematic layout of the PIV apparatus, which was based on a double-pulsed Nd:YAG laser. A liquid seeder with 10 Laskin nozzles was used to atomize vegetable oil into neutrally buoyant micrometre-sized droplets $(1-3 \mu \mathrm{m})$. The

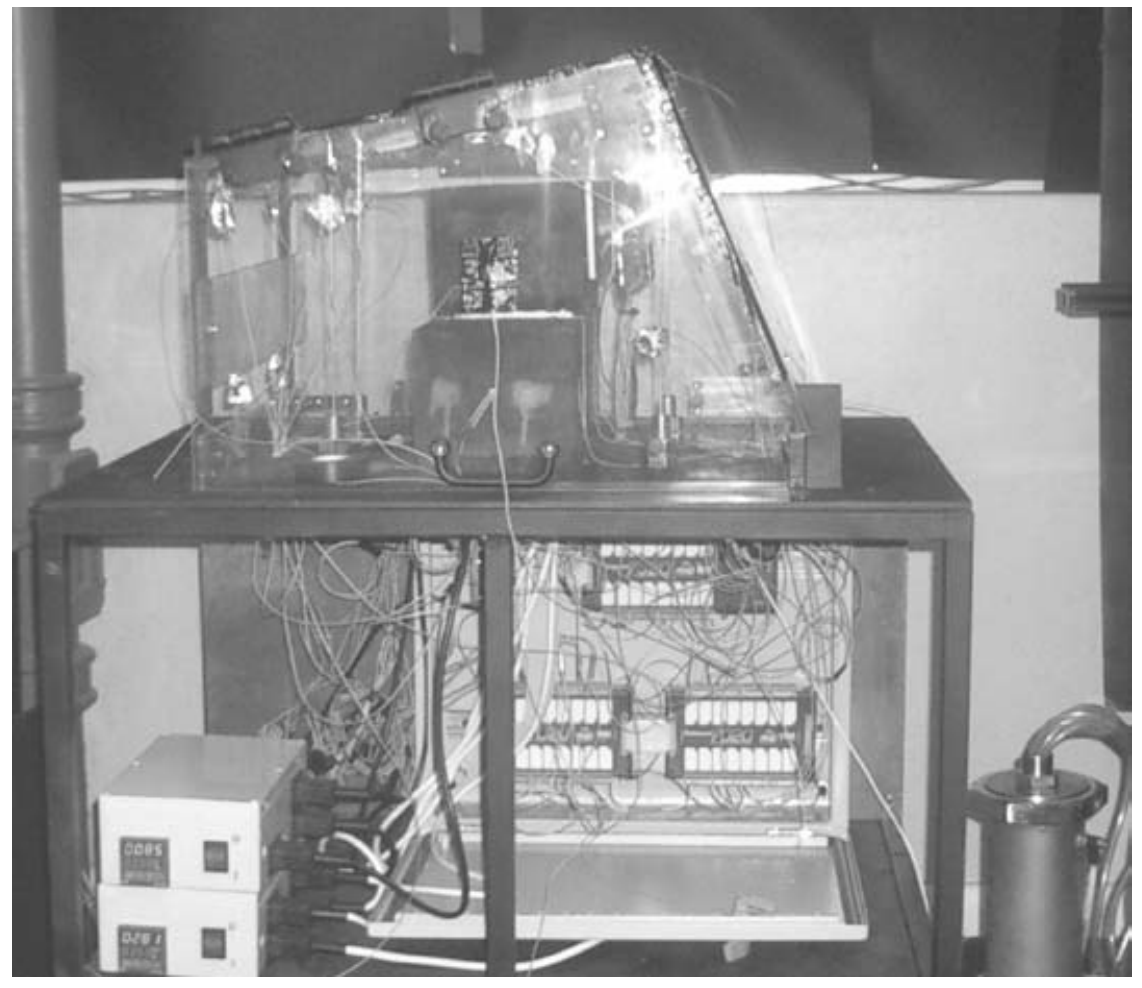

Fig. 6 View of the instrumented laboratory model 


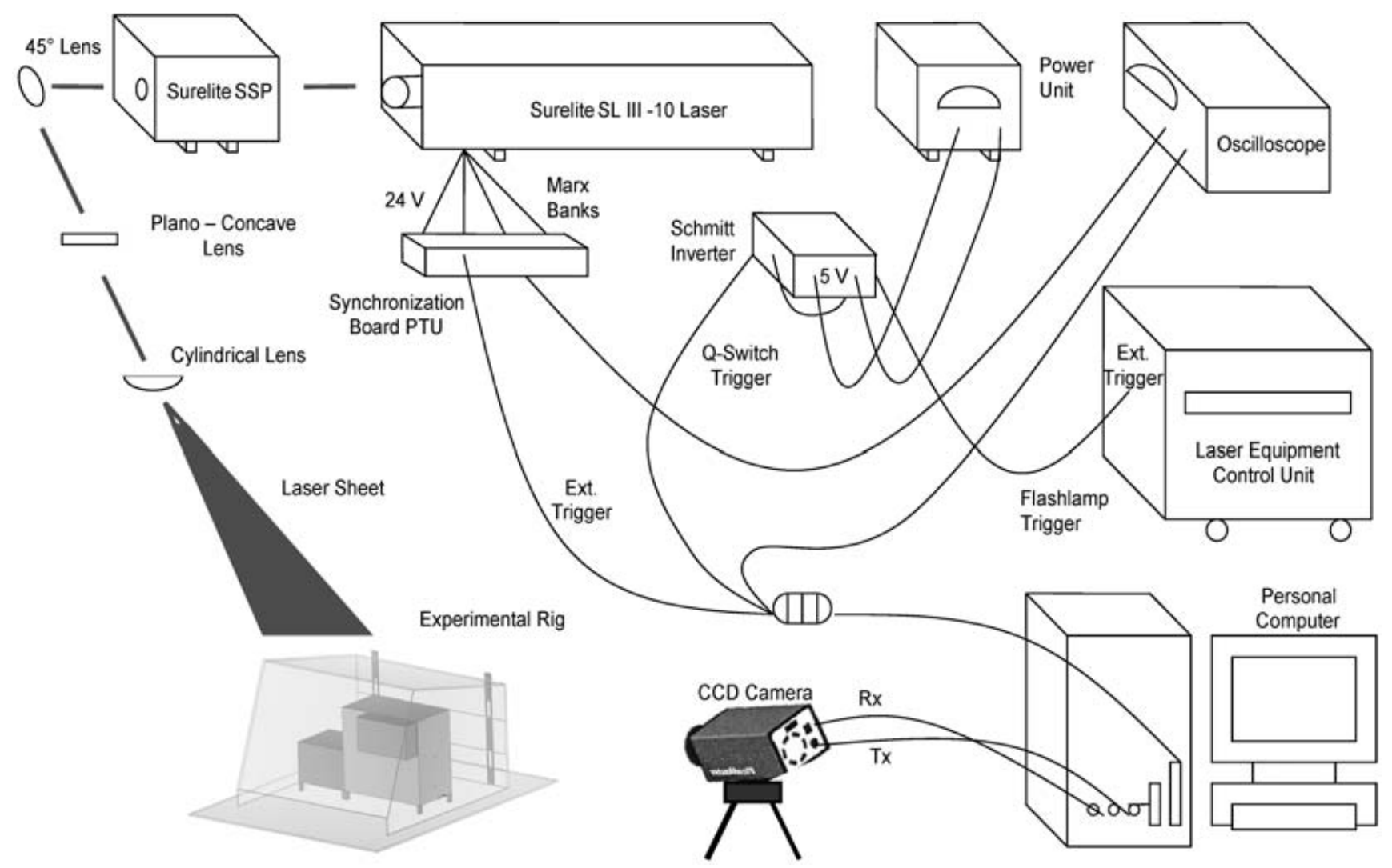

Fig. 7 Schematic layout of PIV apparatus set-up for experimental analysis [7]

particles were produced and released with controllable and repeatable size and density. The low flash point and decomposition temperature of the oil were not found to be a problem as the particles were confirmed to follow the flow streamlines by calculation and visual observation [8].

Owing to very low flow velocity, small seeding size, and the limitations of the illumination system (laser unit of single head type with a maximum limit on pulse time separation), the sizes of the field of view were inevitably small, typically $35 \mathrm{~mm}$ by $30 \mathrm{~mm}$. Measurements of velocity components were repeated and the ensemble averaged.

\subsection{Simulation with the proposed transient procedure}

The CFD analysis was based on the conjugate heat transfer simulation of five solid models (engine, gearbox, turbocharger, compartment, and an insulating pad located between the engine and turbocharger) and one fluid model (air domain). All three modes of heat transfer, including radiation, were solved. Flow turbulence effects were calculated with the standard version of the $\kappa-\varepsilon$ model with law of the wall functions [9]. A structured Cartesian mesh was defined and different grids were prepared for each CHT domain.

The calculations were based on approximately 330000 cells for the mesh discretizing the flow field and a total of 67000 cells for the solid models (average cell size of $15 \mathrm{~mm}$ ). The control volume of the computational chamber containing the underbonnet model was configured with a total pressuretype boundary set in correspondence to the bottom surface and an outlet mass flowrate of $0.001 \mathrm{~kg} / \mathrm{s}$ imposed at the top surface (practically prescribing still ambient conditions). Model surfaces not exchanging thermal information (e.g. the base of the model representing the vehicle undertray) were specified as adiabatic boundaries.

The SWITCH computational procedure was implemented for the numerical solution, having been recognized as the most effective and computationally efficient variant. Based on the preliminary evaluation of the various solver parameters and considering the compromise between accuracy and CPU runtime, the pseudo-transient methodology was set up as follows.

1. Fast transient computations. These were set to solve only the temperature of the coupled fluid and solid domains and the effects of radiation 
heat transfer for a total of 20 steps, each 1 second in duration.

2. Full steady state computations. These were set to solve for 50 iterations all the governing equations for the fluid domain, except for the temperature, and no equations for the solid domains. The flow field was solved with the $\kappa-\varepsilon$ model and the momentum and continuity equations were coupled with the SIMPLE algorithm. Solution stability and convergence were enhanced by the multigrid iterative solver scheme available in VECTIS [6]. The effects of radiation heat transfer were calculated based on the model surfaces being at fixed temperatures (calculated during the previous fast transient run).

3. Number of methodology cycles. A total of 90 cycles was set to cover the 30 minutes of interest, since the progress in time of each cycle was 20 seconds.

\subsection{Data comparison}

The PIV measurements and the CFD predictions gave an insight into the evolution of the flow patterns and surface temperature distribution in the model during the investigated thermal process. In general, it was observed that the movement of the ambient air from both the front and the rear bottom openings into the compartment resulted in a horizontal flow just above the base level. The average inlet air velocities were very similar at both apertures and halved in intensity during the 30 minute timeframe. Within the enclosure, the maximum air velocity was predicted next to the turbocharger block surfaces, directed towards the upper boundaries of the compartment. The turbocharger block temperature was predicted to reduce from 355 to $170^{\circ} \mathrm{C}$ over the investigated timeframe and, accordingly, the maximum air velocity was calculated to reduce from 0.45 to $0.3 \mathrm{~m} / \mathrm{s}$. Above the blocks, re-circulating vortices were observed. Such flow patterns developed owing to the geometric shape of the compartment walls bounding the flow domain and were found to remain nearly constant in diameter (approximately $50 \mathrm{~mm}$ ) throughout the simulated timeframe. The air flowing in the upper layers of the underbonnet were predicted to discharge to ambient from the top front aperture, with a flowrate that nearly halved in 30 minutes.

The temperature of the engine, gearbox, and turbocharger blocks were observed to reduce respectively by approximately $6.4,4.5$, and 51 per cent, over the investigated 30 minutes. The cooling rate was relatively constant for all the blocks and for the compartment surfaces. Temperature measurements for representative block surfaces and airplanes, and
PIV results for selected airflow regions, are compared with CFD predictions in Figures 8 to 12.

\subsubsection{CFD versus thermal measurements}

The temperature evolution of the turbocharger block and of the flow field across a plane located in front and parallel to the engine block are shown in Fig. 8. The experimental data are plotted as the average temperature value among all thermocouple measurements, while the CFD data represent the average of the temperature predictions extracted at the same coordinate locations.

Figure 8(a) demonstrates that the transient procedure was successful in predicting correctly the cooling rate of the block; a very good agreement between measured and predicted temperatures was obtained. The slight discrepancy observed from $900 \mathrm{~s}$ onwards was thought to be attributed to the small uncertainty in the specification of the heat capacity of the blocks in VECTIS. The comparison of the air temperature distribution, as shown in Fig. 8(b), demonstrates that the pseudo-transient procedure was able to provide a reasonably accurate flow field prediction. A quantitative discrepancy of 4.3 per cent, on average, was observed between the two sets of data.

Temperature measurements and CFD temperature predictions for the top surface of the compartment (vehicle bonnet) are presented at 600 and $1200 \mathrm{~s}$ (Figs 9(a) and (b) respectively). The data were processed with a specifically written Matlab ${ }^{\mathrm{TM}}$ program, which interpolated the experimental and numerical data points at exactly the same spatial coordinate locations, providing two-dimensional contour maps.

Although the simulation slightly overpredicted the temperatures, the overall agreement between the two data sets was very satisfactory, with the temperature trends across the surface well predicted. Experimental and CFD data points were also plotted (right-hand side of Fig. 9), with an error bar representing the overall accuracy limit of the instrumentation used (approximately \pm 5 per cent), and as an average value for the coordinate cell corresponding to the thermocouple location and the surrounding cells, with maximum and minimum limits (approximately \pm 3 per cent). The uncertainty of the extracted CFD values was due to the volume meshing of the air domain.

The plots confirmed the good quality of the temperature correlation since the error bands overlapped. The average discrepancy was 4.2 per cent after 10 minutes and 5.2 per cent after 20 minutes, demonstrating consistent time accuracy of the implemented calculation methodology. 
(a)

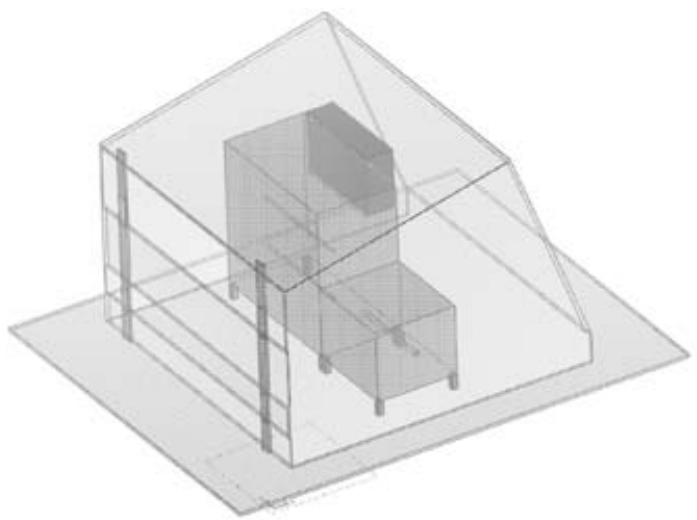

(b)
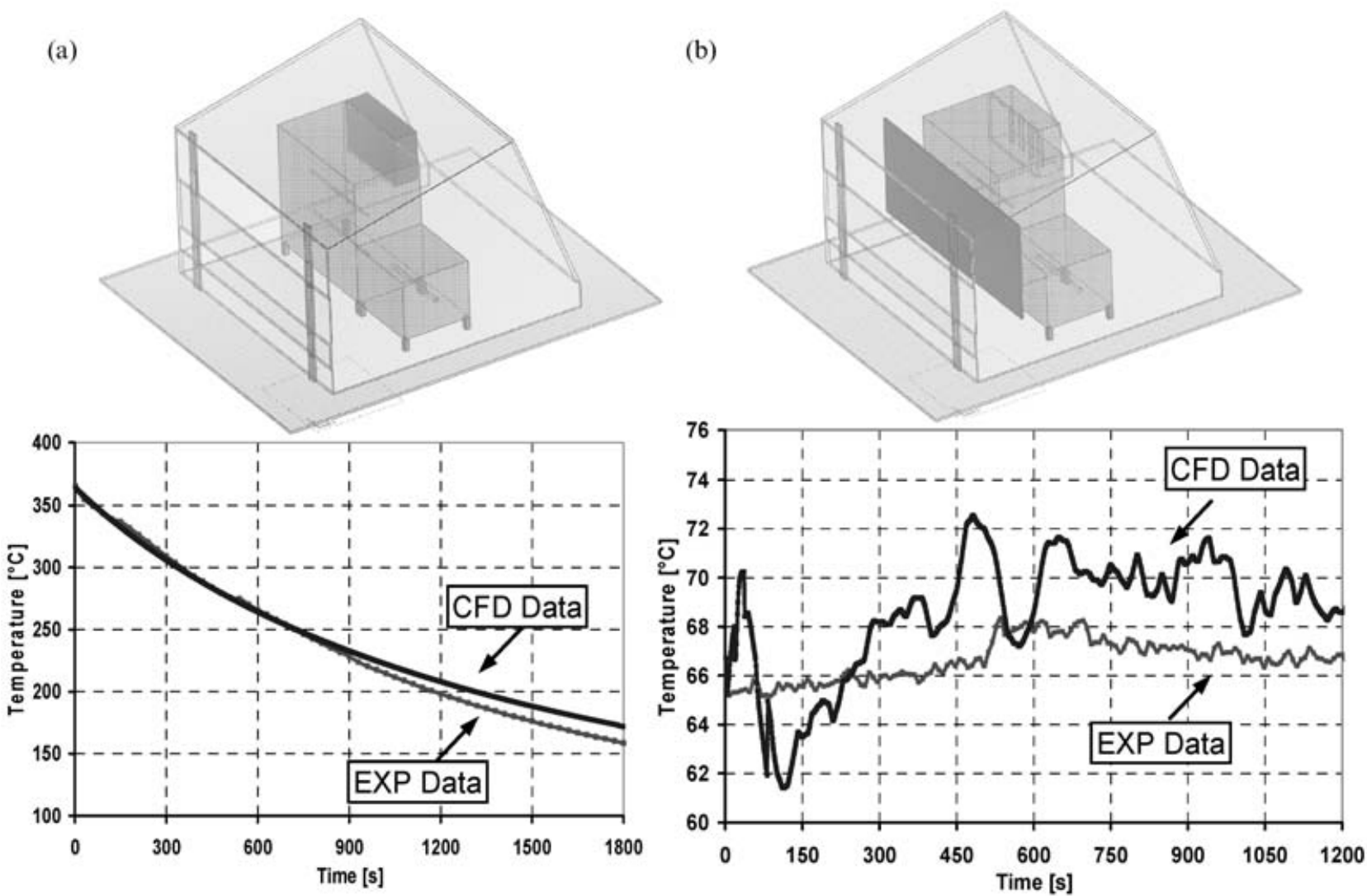

Fig. 8 Comparison of average temperature variation in transient (cooling) conditions for the turbocharger block and the front air plane across the underbonnet

\subsubsection{CFD versus PIV measurements}

Figures 10 (a) and (b) display the predicted flow structure at the top of the turbocharger block (on a vertical plane located at the centre of the surface) at 30 seconds and 1 minute. In each representation, the PIV results (left frame) are compared with the corresponding flow simulated with VECTIS (right frame). The velocity scale for the PIV measurements is given on the right-hand side of each subfigure.

Both the experimental and the computational solutions were clearly characterized by the convective air stream rising from the side of the block surface. The airflow in the compartment attained its maximum strength in this region, which was overpredicted by 6 per cent after 30 seconds and 8.2 per cent after 1 minute. The discrepancies were within the uncertainty of the experimental apparatus for detecting very small velocities. It is necessary to point out that the vertical velocity vectors shown in the CFD frame in fact have their origins outside the frame, and therefore they should be discarded when comparing the two sets of results. Though there were only very limited numbers of vectors present in the
CFD frame, the predicted flow direction matched well qualitatively with the measurement.

The flow field correlation for a vertical plane above the gearbox block, in the vicinity of the side surface of the engine, is presented in Fig. 11(a). The bulk of the airflow was driven both by the air density difference, due to the heat convected by the gearbox, and by the vertical temperature gradient developing on the side of the engine. The flow patterns are compared after 5 minutes of cooling. The flow magnitudes and directions were satisfactorily predicted by VECTIS. The average air velocity was estimated with a discrepancy of $0.021 \mathrm{~m} / \mathrm{s}$, which corresponded to an 18 per cent difference with PIV data.

The measured and predicted average flow velocities of the region in the time domain are compared in Fig. 12. The agreement between the two data sets improved after the first 10 minutes of the simulation and it was always found to be within the uncertainty limits associated with the two techniques.

The flow predictions at the top of the compartment were particularly challenging because the convective stream was characterized by a clockwise circular motion. Figure 11(b) presents an airflow 


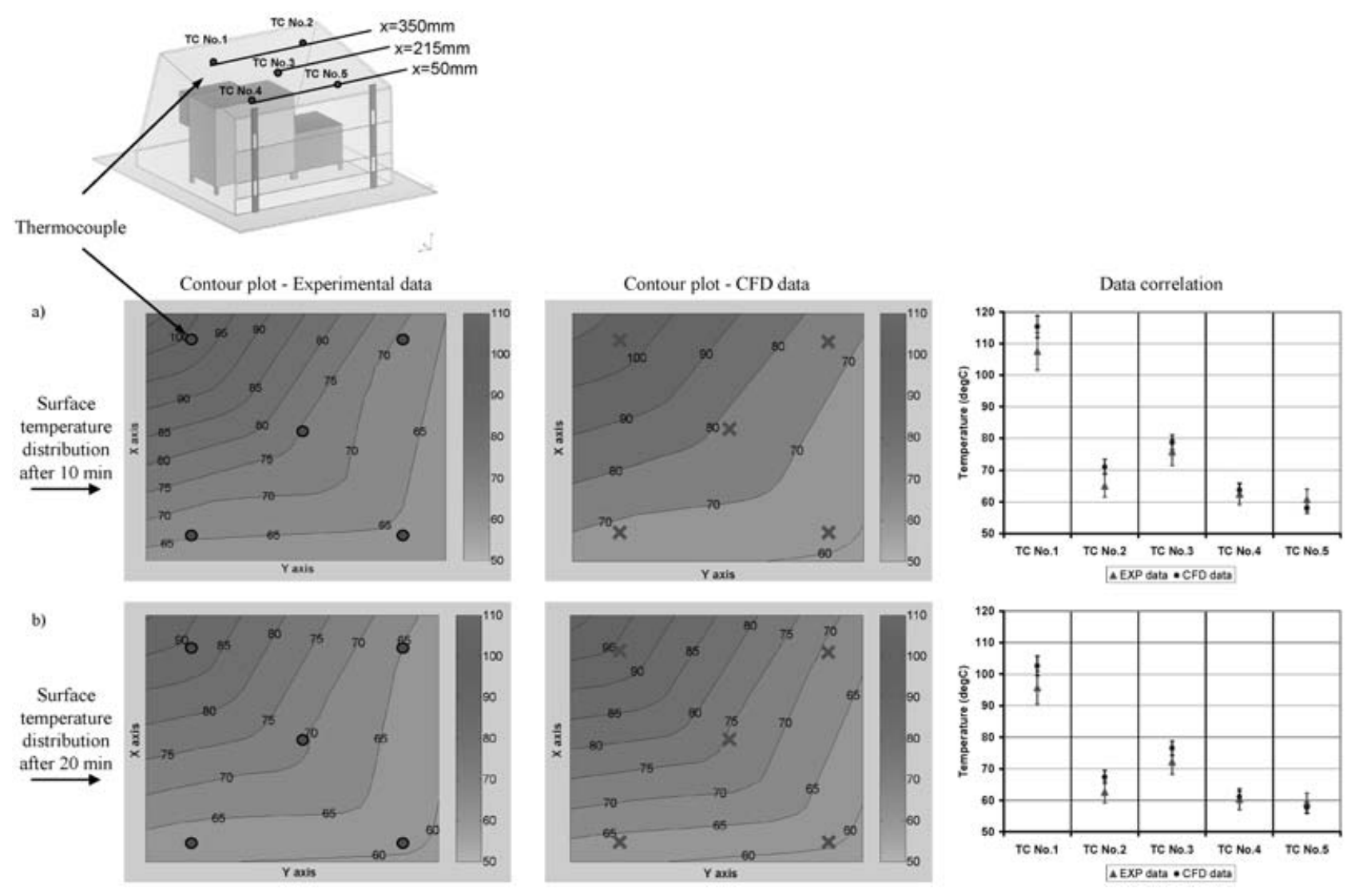

Fig. 9 Thermal correlation for the engine compartment's top surface (vehicle bonnet), with temperatures in degrees Celsius
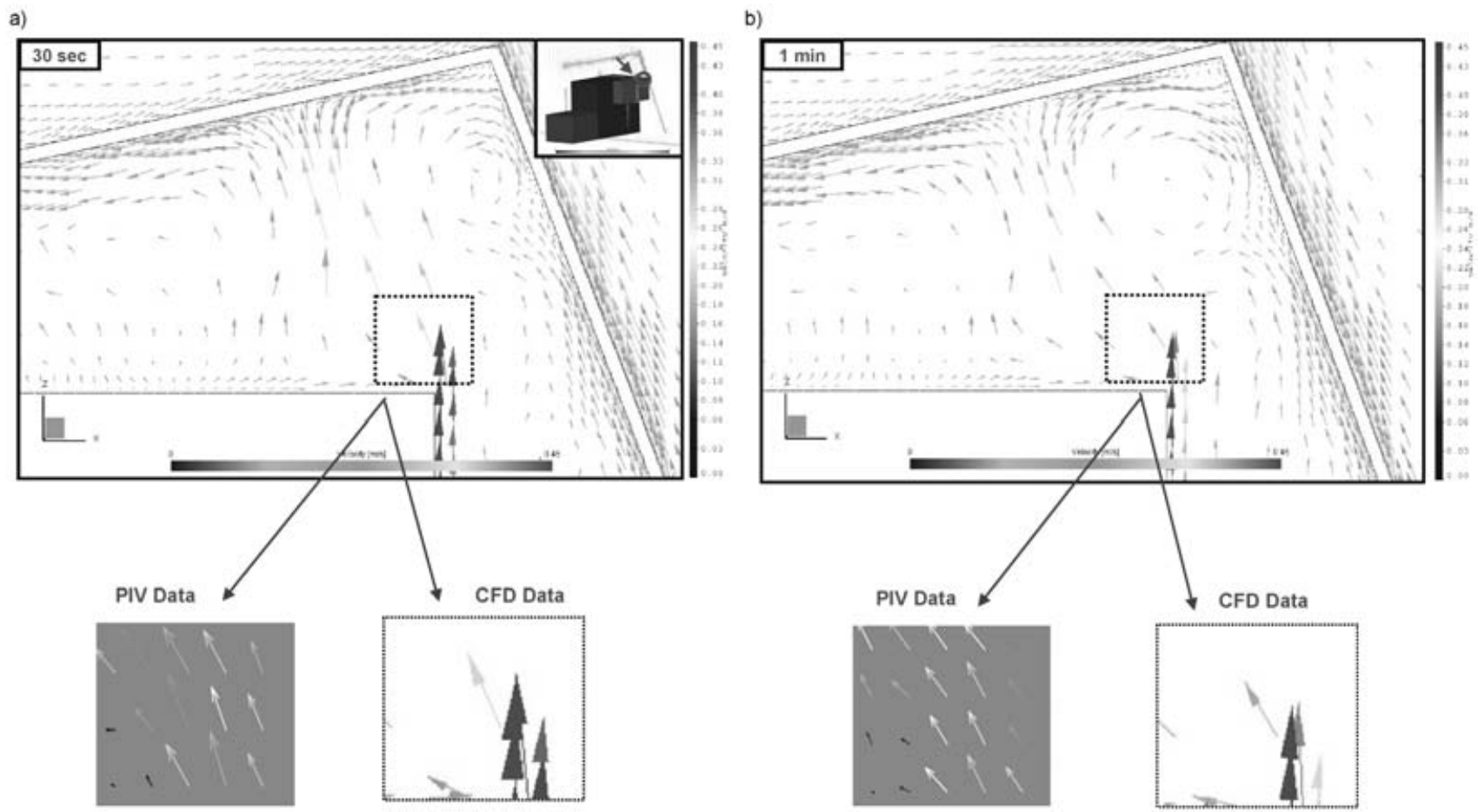

Fig. 10 CFD airflow pattern predictions above the turbocharger block compared with PIV measurements after $30 \mathrm{~s}$ and $1 \mathrm{~min}$ 
a)

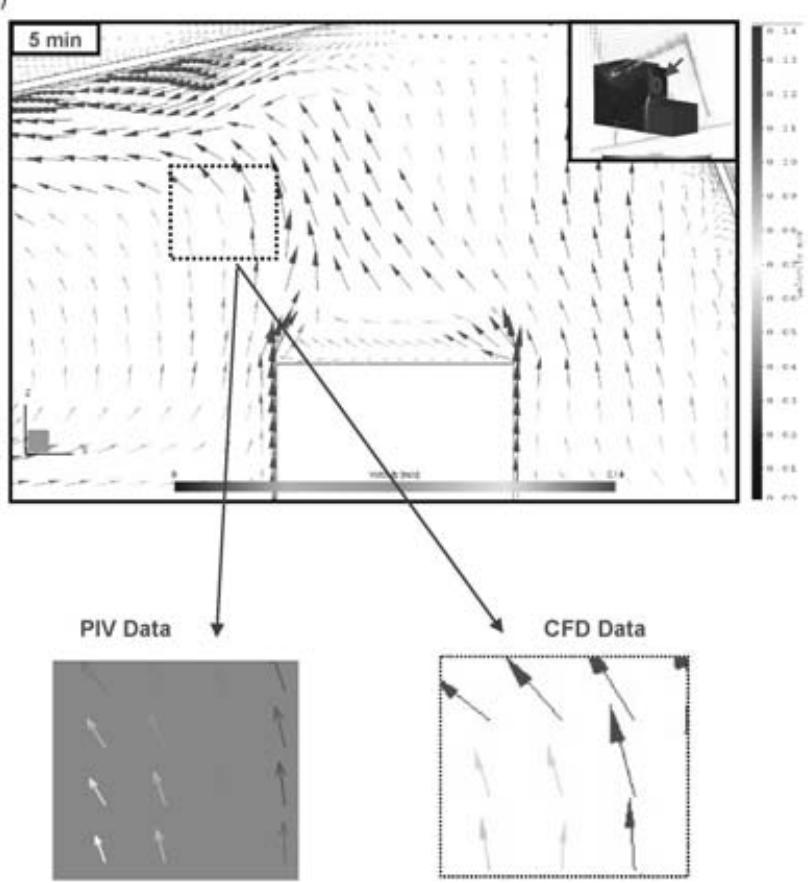

b)

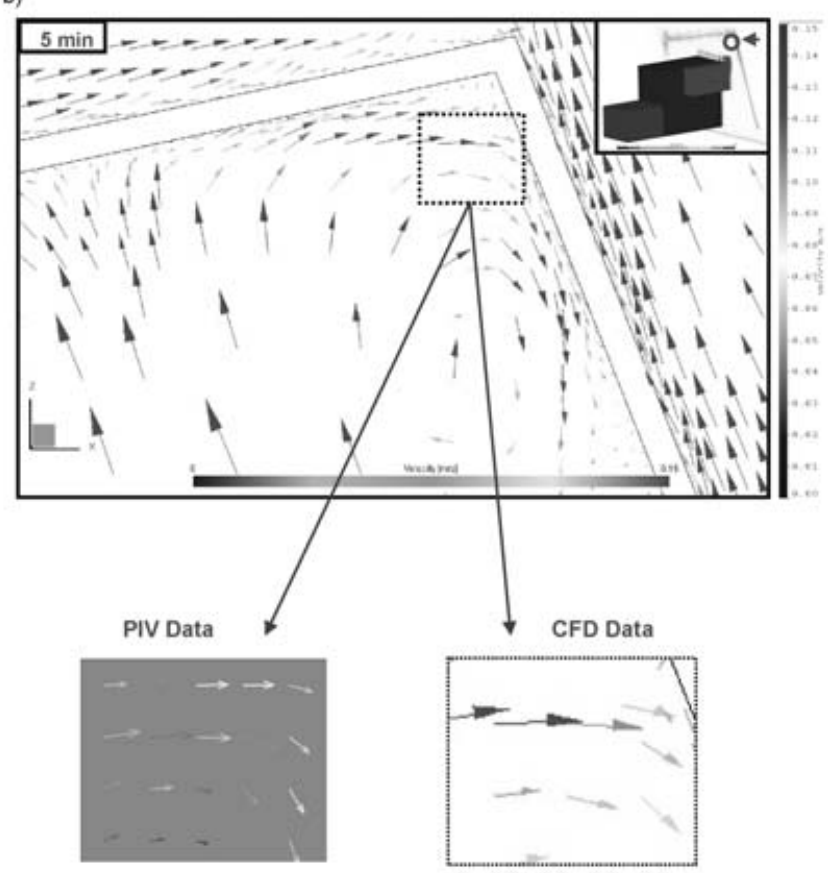

Fig. 11 CFD airflow pattern predictions above (a) the gearbox block and at (b) the top of the compartment compared with PIV measurements after 5 min

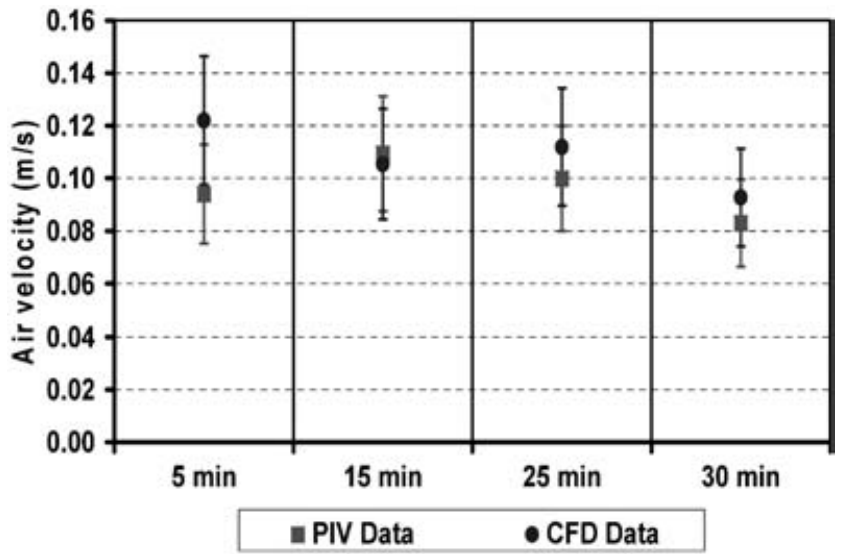

Fig. 12 Correlation of the average velocity vector in the $x z$ flow region above the gearbox block

comparison 5 minutes from the start of the analysis. The flow motion was well predicted, but the average quantitative discrepancy between the correlated velocity magnitudes was of about 50 per cent, with CFD overpredicting the measurements. The numerical error cannot be entirely owed to the procedure employed for the computation, as high discrepancies were also observed when simulating the underbonnet model in steady state conditions, from which the simulation was set to re-start [7]. It is believed that the accuracy of the flow predictions would improve by increasing the density of the air mesh and by implementing a turbulence model more suited to natural convection than the $\kappa-\varepsilon$ model. Increasing the length of the unfrozen flow periods or the number of methodology cycles carried out by the implemented calculation procedure would not compensate for the underlying shortcomings of the natural convection prediction.

\subsection{Time saving}

The average time taken to simulate 1 second of the thermal process with the proposed calculation methodology was approximately 5 minutes (Pentium ${ }^{\circledR}$ 4, $2.40 \mathrm{GHz}, 1 \mathrm{~Gb}$ RAM). A typical transient simulation, performed on the same hardware unit, took approximately 60 minutes (with a $0.001 \mathrm{~s}$ timestep). It is therefore inferred that the application of the SWITCH methodology can provide a total CPU runtime saving of about 90 per cent compared with a standard transient simulation in VECTIS.

\section{CONCLUSIONS}

A computation procedure was proposed for efficiently modelling transient natural convective flows with CFD. The procedure uses a unique combination 
of CFD solver parameters to overcome the computationally challenging problem of thermally solving coupled fluid and solid domains in time-marching mode and for long periods of physical time. Two variants were tested, both of which operated on the basis of pausing the airflow transport solution during the transient simulation. The final established pseudotransient solution method operated on the basis of steady state updates, with a frozen heat balance solution between the thermally conjugated solid domains.

The procedure was proved to be successful in providing a detailed time-dependent flow and thermal simulation of a simplified vehicle underbonnet model prescribed under thermal soak conditions. Such computation would not have been feasible with a standard, fully conservative transient solution, owing to the enormous demands it poses on the computing hardware. The devised methodology resulted in a reduction of over 90 per cent in the CPU runtime.

It is envisaged that the application of the methodology to the simulation of full-scale engine bays will lead to the early identification of potential thermal risks, enabling engineers to improve both vehicle system integrity and performance at reduced time and costs.

\section{ACKNOWLEDGEMENTS}

The authors would like to thank Ricardo UK for supporting this work and for permission to publish the results.

\section{REFERENCES}

1 Bancroft, T. G., Sapsford, S. M., and Butler, D. J. Underhood airflow prediction using VECTIS coupled to a 1-D system model. In Proceedings of the 5th Ricardo Software International Users Conference, Shoreham-by-Sea, UK, 2000.

2 Skea, A., Jolliffe, A., Harrison, R., Jones, M. R., Cardani, P., and Smith, L. Integrated underbonnet thermal management simulation. In Proceedings of the 6th SAE VTMS Conference, Brighton, UK, 2003, paper C599/074/2003.

3 Asghari, T. A. A transient thermal analysis using a simplified heat transfer coefficient model. In International Symposium on Advanced packing materials: processes, properties and interfaces, Georgia, USA, 2001, pp. 366-371.

4 Srebric, J., Chen, Q., and Glicksman, L. R. Validation of a zero-equation turbulence model for complex indoor airflows. ASHRAE Trans., 1999, 105(2), 414-427.

5 Gortz, S. and Moller, J. Evaluation of the recursive projection method for efficient unsteady turbulent CFD simulations. In Proceedings of the 24th International Congress of the Aeronautical Sciences, ICAS, Yokohama, Japan, 2004.

6 Ricardo Software. VECTIS - computational fluid dynamic software, Theory Manual, Ricardo UK Ltd, 2004.

7 Franchetta, M., Suen, K. O., Williams, P. A., and Bancroft, T. G. Investigation into natural convection in an underhood model under heat soak condition. SAE paper 2005-01-1384, 2005.

8 Franchetta, M. Experimental and transient computational fluid dynamic analysis of vehicle underhood in heat soak. PhD Thesis, University College London, 2006.

9 Launder, B. E. and Spalding, D. B. The numerical computation of turbulent flow. Computer Methods in Appl. Mechanics and Engng, 1974, 3, 269-289. 